\title{
Mixing Performance of Inner Baffle in Shear Thinning Fluid
}

\author{
Haruki FuruKawa ${ }^{*}$, Yoshito MizUnO, and Yoshihito KATO \\ Graduate School of Life Science and Applied Chemistry, Nagoya Institute of Technology, Gokiso-cho, Showa-ku, Nagoya, 466- \\ 8555 , Japan
}

\begin{abstract}
A mixing performance of an inner baffle was investigated in shear thinning fluid. The inner baffle was placed with a clearance between baffle and vessel wall. A traditional two-bladed paddle impeller was used. Four planar baffles were vertically placed in two ways: 1) standard baffle condition 2) inner baffle condition. The standard baffle condition was that the baffle was placed on the mixing vessel wall. A mixing pattern was visualized by decolorization method based on an oxidation-reduction reaction with sodium thiosulfate and iodine. Flow field for shear thinning fluid in mixing vessel was measured by particle image velocimetry and was shown as stream line. A pair of isolated mixing regions (IMR) like doughnut-ring formed above and below the impeller under standard baffle condition. However, IMR dissipated under inner baffle condition. As a result, a mixing time under the inner baffle condition was drastically decreased. This study suggested that inner baffle promoted mixing in shear thinning fluid.
\end{abstract}

\section{Introduction}

Agitation and mixing are used in chemical, polymer and pharmaceutical industries. These industries sometimes treat high viscous Newtonian fluid and non-Newtonian fluid. Mixing in these fluids is operated under laminar region. Laminar mixing using a traditional paddle impeller is difficult because an isolated mixing region (IMR) like doughnut-ring appears above and below the impeller (Metzner and Taylor, 1960; Lamberto et al., 1996). The IMR causes mixing to take a long time. Therefore, many kinds of mixing equipment and methods were developed to promote laminar mixing. A large impeller like helical ribbon impeller is used to improve laminar mixing (Carreau et al., 1976; Furukawa et al., 2013; Kato et al., 2015; Kato et al., 2018; Mihailova et al., 2018). Another method is unsteady mixing method (Murakami et al., 1980; Kato et al., 2005). However, the large impeller requires a large rotational torque and the unsteady mixing requires a complex equipment.

In our previous study (Furukawa et al., 2018), we found that a baffle placed with a clearance between the baffle and the mixing vessel promoted laminar mixing in Newtonian fluid. This mixing method just requires a simple equipment and the rotational torque is low. In this study, we investigated an effect of the baffle placed with a clearance on mixing performance in shear thinning fluid.

\section{Experimental Setup and Method}

The experimental setup used in this study was shown in Figure 1. The cylindrical mixing vessel had flat bottom.
The mixing vessel was made of acrylic resin and transparent. The inner diameter $T$ of the mixing vessel was $0.185 \mathrm{~m}$. The mixing vessel was placed inside a rectangular outer acrylic tank filled with tap water in order to minimize optical distortions caused by the curvature of the tank. The liquid height $H$ was equal to $T$. The impeller was paddle type having flat blade. The number of blade $n_{\mathrm{P}}$ was 2 . The diameter of the impeller $d$ was $0.5 T$ and the height of the impeller $b$ was $0.2 d$. The width of a baffle $B_{\mathrm{W}}$ was $0.1 T$ and number of the baffle $n_{\mathrm{B}}$ was 4 . The baffle was vertically inserted to the vessel at every $90^{\circ}$ angle. The clearances $C_{\mathrm{B}}$ between impeller blade tip and the baffle were $0.05 T$ and $0.15 T$. The condition that the baffle was placed at $C_{\mathrm{B}}=0.05 T$ was called inner baffle condition in this study. Otherwise, the condition that the baffle was set on the vessel wall at $C_{\mathrm{B}}=0.15 T$ was called standard baffle condition.

Carboxymethylcellulose (CMC, FUJIFILM Wako Pure Chemical Corporation) aqueous solution was used as shear thinning fluid in this study. The concentrations of the $\mathrm{CMC}$ aqueous solution were $2.0,3.0$ and $4.0 \mathrm{wt} \%$. The rheology property was measured with rheometer (HAAKE Vicotester VT550, Thermo Fisher Scientific Inc.). Figure 2 showed the rheological property of each of the CMC aqueous solution. The power law model correlated these rheological properties. The power law model is given by

$$
\tau=\alpha \dot{\gamma}^{\beta}
$$

where $\alpha$ and $\beta$ are constants known as the consistency index and power-law exponent, respectively. Table 1 showed $\alpha, \beta$ and density of each of the concentration of $\mathrm{CMC}$ aqueous solution. The mixing Reynolds number Re was calculated by Metzner-Otto method (Metzner and

\footnotetext{
* Corresponding author: furukawa.haruki@nitech.ac.jp
} 


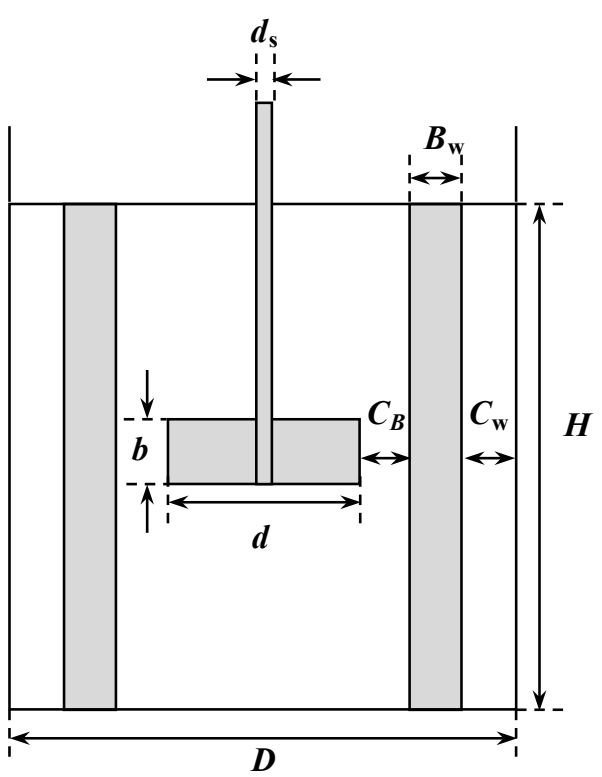

Figure 1. Dimension of the experimental setup

Otto, 1957). The Metzner-Otto constant $k_{\mathrm{S}}$ of the paddle impeller used in this study was 9.89 calculated by correlation for $k_{\mathrm{S}}$ (Kamei et al., 1994).

The mixing performance was investigated by decolorization method based on oxidation-reduction reaction. The oxidation agent was sodium thiosulfate and the reduction agent was iodine. Sodium thiosulfate (FUJIFILM Wako Pure Chemical Corporation) was dissolve in $\mathrm{CMC}$ aqueous solution to obtain the decolorization agent. Iodine (NACALAI TESQUE, Inc.) was dissolved in the potassium iodine (KISHIDA CHEMICAL Co., Ltd.) aqueous solution. The iodinepotassium iodine aqueous solution was added to the CMC aqueous solution to obtain the colorization agent. After the colorization agent colored the working fluid, the decolorization agent was added to the colored working fluid. The ratio of iodine to the sodium thiosulfate was 1 to 1.4. This ratio allowed to determine decolorization easily (Takahashi et al., 1985). The decolorization process was recorded by a digital video camera (HDR-CX420, Sony Marketing Inc.) to observe a mixing pattern.

Particle image velocimetry (PIV) was adopted to measure the velocity profile in the mixing vessel. Figure 3 showed the PIV experimental setup. Passive tracers were nylon particles whose diameter and density were 150-300 $\mu \mathrm{m}$ and $1020 \mathrm{~kg} \mathrm{~m}^{-3}$, respectively. The tracer particles were dispersed in the mixing vessel. The vertical cross section behind the baffle in the mixing vessel was lighted by laser sheet light (Reliant 1000M, LASER PHYSICS). The laser sheet light was emitted to the mixing shaft. The refraction light was captured by the digital video camera. The captured videos were analyzed by using the commercial software FlowExpert2D2C (KATOKOKEN Co., Ltd.). The velocity profile was shown as sream line.

\section{Results and Discussion}

Table 1. Physical properties of non-Newtonian fluid

\begin{tabular}{cccc}
\hline $\mathrm{wt} \%$ & $\alpha\left[\mathrm{Pa} \mathrm{s}^{\beta}\right]$ & $\beta[-]$ & $\rho\left[\mathrm{kg} \mathrm{m}^{-3}\right]$ \\
\hline 2.0 & 1.73 & 0.656 & 1008 \\
3.0 & 5.55 & 0.590 & 1013 \\
4.0 & 19.6 & 0.451 & 1016 \\
\hline
\end{tabular}

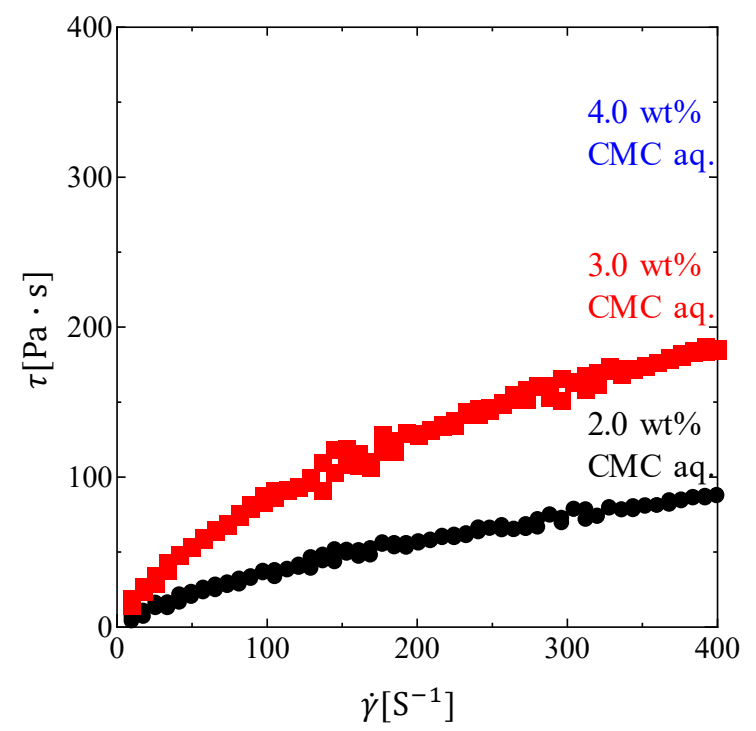

Figure 2. Rheological properties of CMC aqueous solution

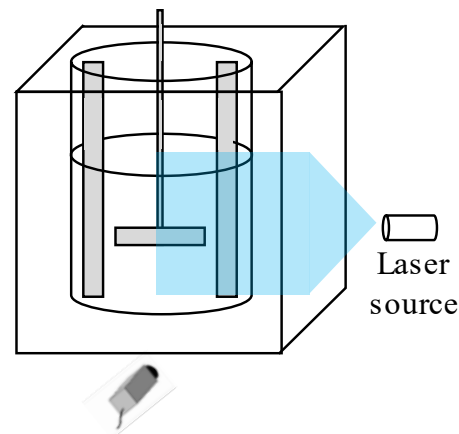

Digital video camera

(a) side view

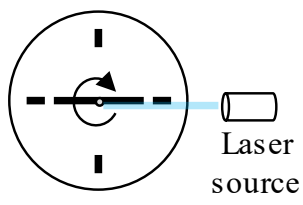

(b) over view
Figure 3. Schematic illustration of PIV experiment

Figure 4 shows the mixing pattern of $3 \mathrm{wt} \% \mathrm{CMC}$ aqueous solution at $C_{\mathrm{B}} / D=0.05$ and 0.15 at $R e=60$. When the baffles were placed at $C_{\mathrm{B}} / D=0.15$, a pair of IMR like doughnut-ring formed above and below the impeller. Otherwise, the IMR was dissipated by placing baffle at $C_{\mathrm{B}} / D=0.05$.

The decolorization experiment was conducted under various $R e$ to investigate the effect of $R e$ on the mixing pattern by placing baffles at $C_{\mathrm{B}} / D=0.05$. Figure 5 shows the mixing pattern under $R e=30$ and 40 . The unmixed region like IMRs was observed below the impeller under both $R e$ at $n t=3000$. However, this unmixed region was dissipated at $n t=5000$. Lamberto et al. (1999), Hahimoto et al. (2009) and Wang et al. (2013) stated that the IMRs like doughnut-ring move toward impeller in radial direction with decreasing $R e$ in Newtonian fluid. It is considered that the IMR also moved toward impeller in this work. Therefore, after unmixed region IMRs formed under both $R e$, the unmixed region was dissipated soon. 


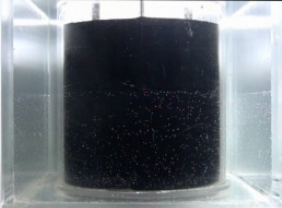

$n t=0$

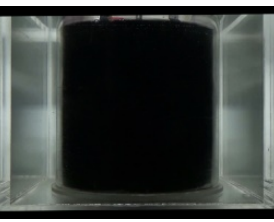

$n t=0$

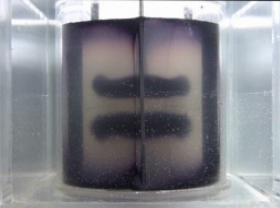

$n t=500$

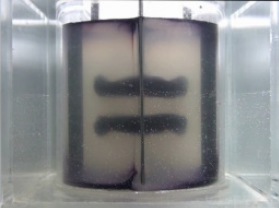

$n t=1000$

(a) $C_{\mathrm{B}} / D=0.15$

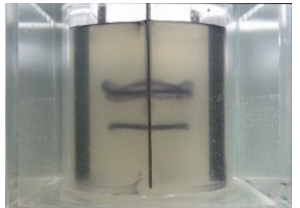

$n t=3000$

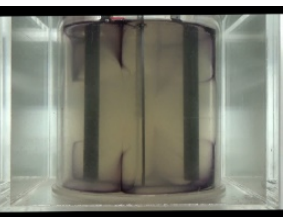

$n t=3000$

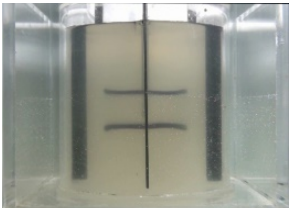

$n t=5000$

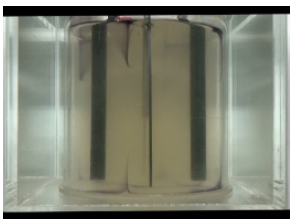

$n t=5000$

(b) $C_{\mathrm{B}} / D=0.05$

Figure 4. Effect of baffle location on mixing pattern in $3 \mathrm{wt} \% \mathrm{CMC}$ aq. solution under $R e=60$

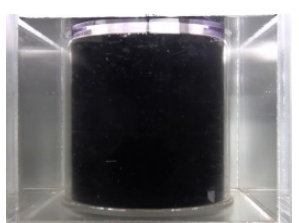

$n t=0$

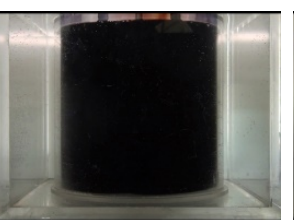

$n t=0$

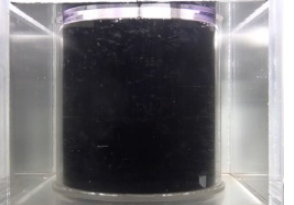

$n t=500$

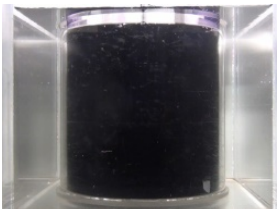

$n t=1000$

(a) $R e=30$

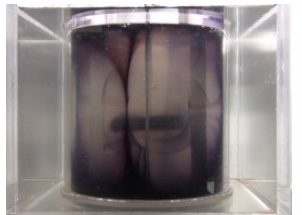

$n t=3000$

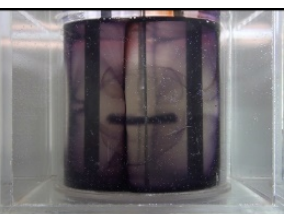

$n t=3000$

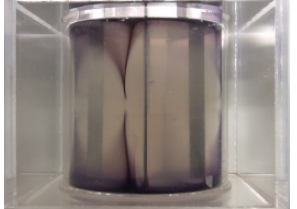

$n t=5000$

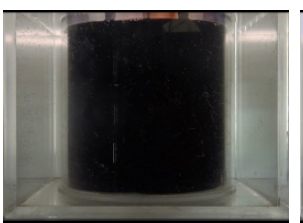

$n t=500$

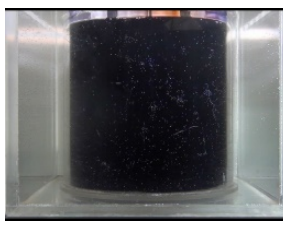

$n t=1000$

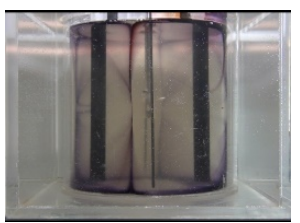

$n t=5000$

(b) $R e=40$

Figure 5. Effect of Reynolods number on mixing pattern in $3 \mathrm{wt} \% \mathrm{CMC}$ aq. solution

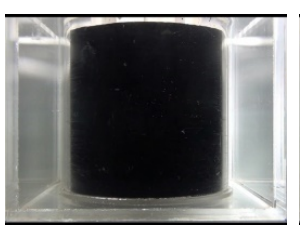

$n t=0$

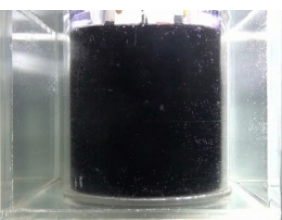

$n t=0$

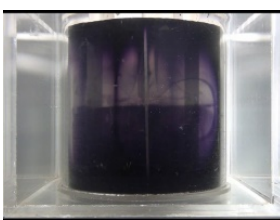

$n t=100$

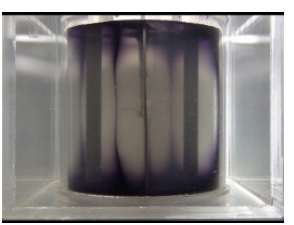

$n t=300$

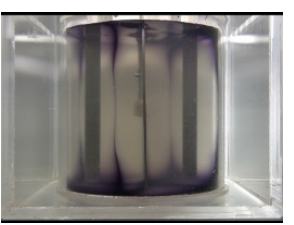

$n t=500$

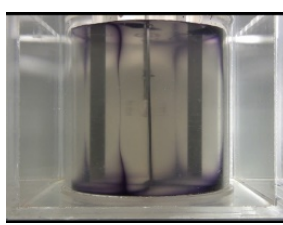

$n t=700$

(a) $2 \mathrm{wt} \% \mathrm{CMC}$ aq. solution

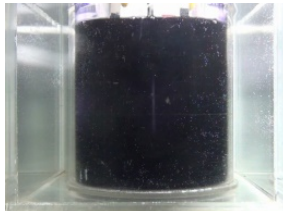

$n t=1000$

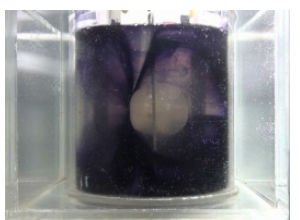

$n t=3000$

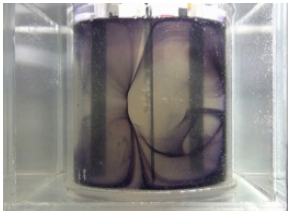

$n t=5000$

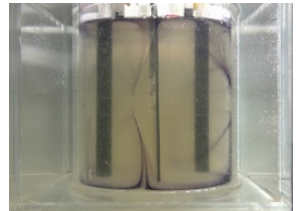

$n t=10000$

(b) $4 \mathrm{wt} \% \mathrm{CMC}$ aq. solution

Figure 6. Mixing pattern in various concentration of $\mathrm{CMC}$ aq. solution.

The effect of concentration of CMC aqueous solution on mixing pattern was investigated. Figure 6 shows the result of decolorization experiment under various concentration of CMC aqueous solution under $R e=60$.
The placing baffle at $C_{\mathrm{B}} / D=0.05$ promoted under each of concentration of CMC aqueous solution.

The velocity profile in the mixing vessel was investigated by PIV. Figure 7 shows the stream line per 


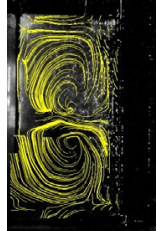

$1 / 8 T$

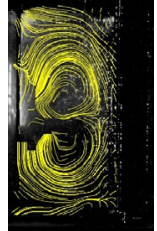

$3 / 8 T$

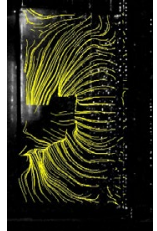

$1 / 8 T$

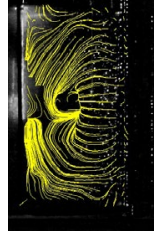

$3 / 8 T$ (a) $C_{\mathrm{B}} / D=0.15$

(b) $C_{\mathrm{B}} / D=0.05$

Figure 7. Stream line under $R e=70$

1/8 impeller rotation $T$ and 3/8T. The impeller was parallel to the vertical cross section at $1 / 8 T$ and vertical to that at $3 / 8 T$. This stream line was taken under $R e=70$ in $2 \mathrm{wt} \%$ CMC aqueous solution. Fig. 7(a) shows the stream line at $C_{\mathrm{B}} / D=0.15$. Two secondary circulations formed and made IMRs like doughnut-ring similarly to no baffle condition. The baffle under standard baffle condition did not improve laminar mixing in a shear thinning fluid. Otherwise, Fig. 7(b) shows the stream line when the baffles was placed at $C_{\mathrm{B}} / D=0.05$. Two secondary circulations unformed partially under this baffle location because setting the baffle at $C_{\mathrm{B}} / D=0.05$ prevented the secondary flow from turning over like ring. Therefore, IMRs like doughnut-ring above and below impeller unformed to improve mixing state in shear thinning fluid.

\section{Conclusion}

Decolorization experiments and PIV experiments were conducted to investigate the effect of inner baffle on mixing performance in shear thinning fluid. Placing baffle at $C_{\mathrm{B}} / D=0.05$ improves mixing state compared to placing baffle at $C_{\mathrm{B}} / D=0.15$ called standard baffle condition. It is caused that the baffle at $C_{\mathrm{B}} / D=0.05$ prevents secondary circulation from forming vortices like ring.

It is considered that the installing baffle has no performance of improving laminar mixing. However, this study shows the installing baffle promotes the laminar mixing in shear thinning fluid by changing the location of baffle.

\section{References}

Carreau, P. J., I. Patterson and C. Y. Yap; "Mixing of Viscoelastic Fluids with Helical-Ribbon Agitators I Mixing Time and Flow Patterns," Can. J. Chem. Eng., 54, 135-142 (1976)

Furukawa, H., S. Ohtani S., Y. Kato and Y. Tada; "Effects of Location of Baffle and Clearance between Baffle and Vessel Wall on Isolated Mixing Regions," 51, 29-32 (2018)

Furukawa, H., Y. Kato, T. Kato and Y. Tada; "Power Correlations and Mixing Patterns of Several Large Paddle Impellers with Dished Bottoms," J. Chem. Eng. Japan, 46, 255-261 (2013)
Hashimoto, S., H. Ito and Y. Inoue; "Experimental Study on Geometric Structure of Isolated Mixing Region in Impeller Agitated Vessel," Chem. Eng. Sci., 64, 51735181 (2009)

Kato, Y., T. Nemoto, K. Ago, T. Arai, H. Furukawa and Y. Kato; "Mixing Performance of LR500 Impeller for Highly Viscous Fluid," Kagaku Kogaku Ronbunshu, 44, 346-349 (2018)

Kato, Y., S. Ohtani and H. Furukawa; "Characteristics of Power Consumption and Mixing Time of New Large Paddle (HB Type) Impeller," Kagaku Kogaku Ronbunshu, 41, 276-280 (2015)

Lamberto D. J., F. J. Muzzio, P. D. Swanson and A. L. Tonkovich; "Using Time-Dependence RPM to Enhance Mixing in Stirred Vessels," Chem. Eng. Sci., 51, 733-741 (1996)

Lamberto, D. J., M. M. Alvarez, and F. J. Muzzio; "Experimental and Computational Investigation of the Laminar Flow Structure in a Stirred Tank," Chem. Eng. Sci., 54, 919-942 (1999)

Metzner A. B. and R. E. Otto; "Agitation of NonNewtonian Fluids," AIChE J., 3, 3-10 (1957)

Metzner A. B. and J. S. Taylor; "Flow Patterns in Agitated Vessels," AIChE J., 6, 109-114 (1960)

Mihailova O., T. Mothersdale, T. Rodgers, Z. Ren, S. Watson, V. lister, and A. Kowalski; "Optimisation of Mixing Performance of Helical Ribbon Mixers for High Throughput Applications Using Computational Fluid Dynamics," 132, 942-953 (2018)

Takahashi, K., T. Takahata, T. Yokota, and H. Konno; "Mixing of Two Miscible Highly Viscous Newtonian Liquids in a Helical Ribbon Agitator," J. Chem. Eng. Japan, 18, 159-162 (1985)

Wang, S., J. Wu and E. Y. Bong; "Reduced IMRs in a Mixing Tank via Agitation Improvement," Chem. Eng. Res. Des., 91, 1009-1017 (2013) 